\title{
DIVERSIDAD DE CONOCIMIENTO ETNOBOTÁNICO TRADICIONAL EN LA RESERVA DE LA BIOSFERA “EL CIELO”, TAMAULIPAS, MÉXICO
}

\section{DIVERSITY OF TRADITIONAL ETHNOBOTANY KNOWLEDGE AT "EL CIELO" BIOSPHERE RESERVE, TAMAULIPAS, MÉXICO}

\author{
Sergio Guillermo Medellín-Morales ${ }^{1}$, Ludivina Barrientos-Lozano ${ }^{2}$, Arturo Mora-Olivo ${ }^{3}$, Pedro Almaguer- \\ Sierra $^{4}$ y Sandra Grisell Mora-Ravelo ${ }^{5}$
}

\begin{abstract}
Resumen
El objetivo de la investigación fue determinar la riqueza de conocimiento etnobotánico que poseen habitantes de dos comunidades locales. Para ello se realizaron entrevistas al azar al $30 \%$ de los habitantes de ambas localidades (66 personas en total) y dos talleres participativos comunitarios (uno por localidad). Se identificaron 156 taxones útiles correspondientes a 62 familias botánicas. La riqueza de conocimiento (RQZ) en ambas comunidades es similar, observándose un nivel de conocimiento homogéneo en los diferentes grupos etarios de ambas comunidades. Sólo en la comunidad de Alta Cima existe una diferencia significativa en el grupo etario de 41 a 50 años. Quienes conocen más sobre plantas útiles son los hombres (en Alta Cima), mientras que en San José son similares los valores entre hombres y mujeres. La mayoría de las especies útiles son nativas en ambas comunidades, aunque hay un mayor porcentaje de especies exógenas demandadas en San José. El conocimiento empírico de las especies vegetales, el cual persiste hasta el día de hoy, y en forma más notaria en comunidades rurales, es el resultado directo de los usos que el ser humano ha dado esas plantas.

Palabras clave: Reserva de la Biosfera El Cielo, bosque de niebla, conocimiento campesino, etnobotánica.
\end{abstract}

\begin{abstract}
This research aimed to determine richness on ethnobotanical knowledge possessed by inhabitants of two local communities. For this, $30 \%$ of the inhabitants of both localities ( 66 people in total) were randomly interviewed, and two participatory community workshops (one per locality) were performed. We identified 156 useful taxa corresponding to 62 botanical families. Richness on ethnobotanical knowledge (RQZ) in both communities is similar, observing a homogeneous level of knowledge in different age groups of both communities. Only in the Alta Cima community there is a significant difference in the age group from 41 to 50 years. Those who know more about useful plants are men (in Alta Cima), while in San Jose the values between men and women are similar. Most useful species are native to both communities, although there is a greater percentage of exogenous species claimed in San Jose. The empirical knowledge of plant species, which persists to this day, and more notably in rural communities, is the direct result of the uses that humans have given these plants.
\end{abstract}

Key words: El Cielo biosphere reserve, cloud forest, campesino knowledge, ethnobotany.

\section{Introducción.}

En un sitio biodiverso, como el bosque de niebla de la Reserva de la Biosfera El Cielo (RBEC) que contiene el $10 \%$ de la biodiversidad vegetal de México es importante realizar estudios etnobotánicos que ayuden a comprender las formas de manejo, conocimientos y valores que las personas de estas comunidades construyen en torno a ellos.

Los patrones de uso y manejo de la flora en ambientes montañosos suponen procesos de interacción entre los sistemas sociales y los ecosistemas naturales, adaptándose a los ciclos productivos de los mismos, a los nichos ecológicos resultantes de la verticalidad montañosa y a las transformaciones sociales del momento pasando de una generación a otra bajo una dinámica de transferencia y acumulación (Stringer et al., 2006).

Para el caso de la RBEC sólo dos trabajos específicos versan sobre la diversidad etnobotánica en las comunidades de estudio, el de González-Romo \& Gispert (2005) y Pérez-Quilantán et al. (2005). En 1985 al decretarse la RBEC como área natural protegida, se incentivó inconscientemente el uso y manejo de las 
especies no maderables (Gobierno de Tamaulipas \& TNC, 2011).

Se plantea la hipótesis de que los adultos mayores y las mujeres adultas de 20 años y más, poseen los mayores niveles de conocimiento sobre plantas útiles en las comunidades en estudio. El objetivo de la investigación fue determinar las diferencias en el nivel de conocimiento etnobotánico de los habitantes locales por rangos de edad y por sexo.

\section{Materiales y métodos.}

La investigación se realizó en los ejidos Alta Cima y San José, municipio de Gómez Farías (Reserva de la Biosfera El Cielo, RBEC), Tamaulipas (Figura 1). Ambas comunidades se encuentran ubicadas en el cinturón de bosque de niebla de la RBEC, entre los 900 y $1300 \mathrm{msnm}$, en una porción de la Sierra Madre Oriental conocida como Sierra de Guatemala.

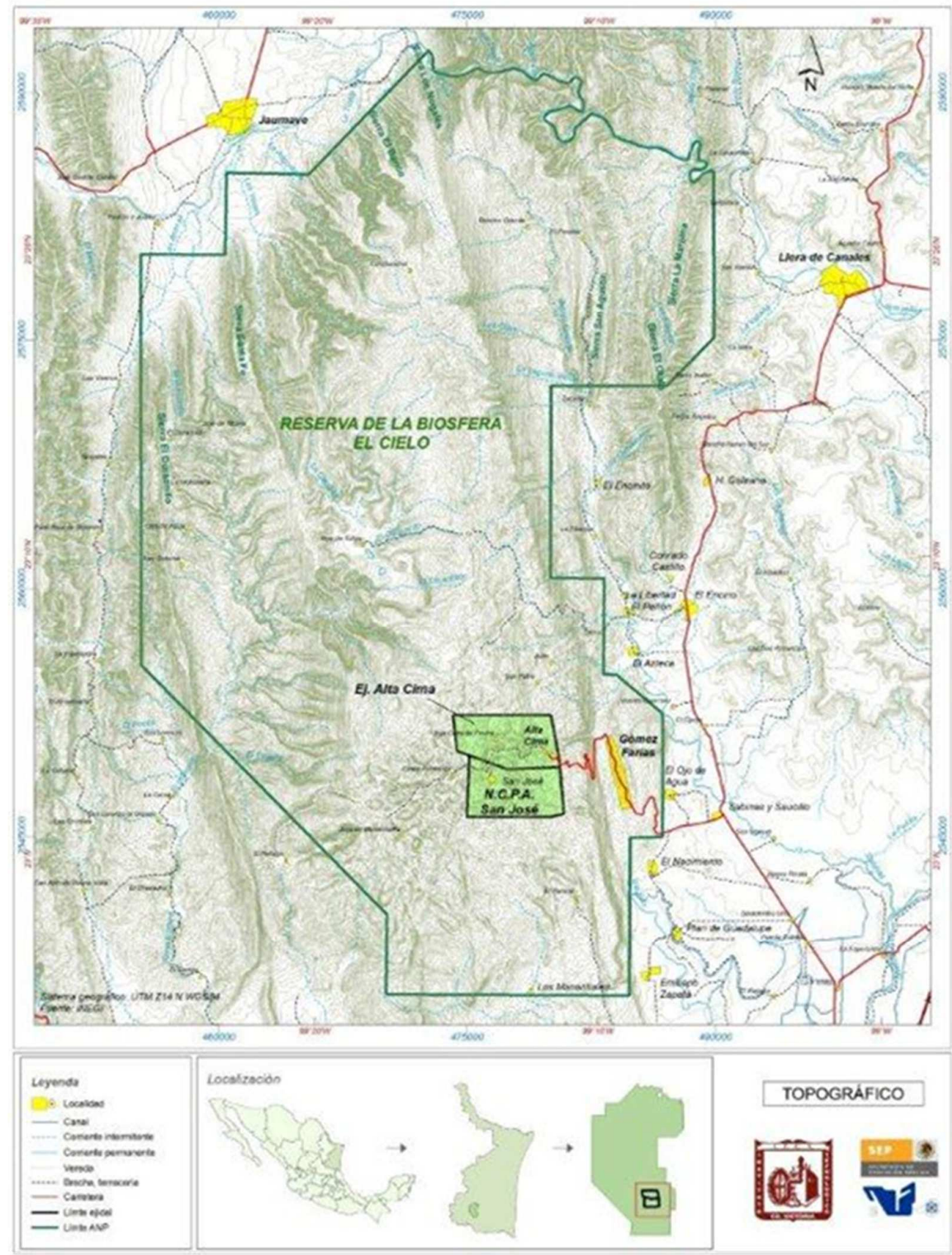

Figura 1. Localización de las comunidades involucradas en la presente investigación, RBEC, Gómez Farías, Tamaulipas, México 
La RBEC tiene una extensión de 144530.51 ha y está situada al suroeste del Estado de Tamaulipas (Gobierno de Tamaulipas \& TNC, 2011).

El sitio presenta influencia de la RH-26 Región Hidrológica Bajo Río Pánuco, con una superficie de 16 226.07 $\mathrm{Km}^{2}$, de ésta se desprende la Cuenca (B) Río Tamesí con una superficie de $15256.50 \mathrm{Km}^{2}$, considerada como uno de los afluentes más importantes del Río Panuco. De ésta última se desprenden la Subcuenca $(\mathrm{Bd})$ con una superficie de 33800 ha (Mendiola, 2010). Geología: Se localiza dentro de la Subprovincia de la Gran Sierra Plegada, la cual presenta un sistema de topoformas que se designan como sierra pliegue o sierra compleja. Esta subprovincia presenta un relieve de valles, existe en ella la unidad que atestigua un tiempo geológico del Mesozoico, en el cual existen afloramientos de rocas sedimentarias (calizas del Cretácico inferior). (Mendiola, 2010).

Ejido Alta Cima se ubica en las coordenadas: $99^{\circ} 11^{\prime} 56^{\prime \prime}$ latitud norte y $23^{\circ} 03^{\prime} 36^{\prime \prime}$ longitud oeste con una altura de $920 \mathrm{msnm}$ (Mendiola, 2010). Presenta clima cálido, subhúmedo con precipitaciones mayores de $2000 \mathrm{~mm}$ anuales (Casas \& Requena, 2005). Considerando la permeabilidad del suelo, el uso actual y la precipitación media, el área del sitio en estudio presenta escurrimientos que varía entre 0.0 y $5.0 \%$ de volumen precipitado (Mendiola, 2010). Se caracteriza por suelo tipo Luvisol crómico (Lc), su limitante es la roca caliza con afloramientos de la misma (Mendiola, 2010). Se presentan dos tipos de vegetación: selva mediana subperennifolia y bosque mesófilo de montaña (bosque de niebla). La selva mediana subperennifolia se desarrolla en laderas poco pronunciadas con suelos ricos en materia orgánica. Alcanza en su estrato arbóreo los $25 \mathrm{~m}$ de altura y tiene especies como Sapindus saponaria L. (jaboncillo), Nectandra salicifolia (Kunth) Nees (aguacatillo), Guazuma ulmifolia Lam., (aquiche) y Quercus spp., (encino). El bosque mesófilo de montaña (bosque de niebla) crece en las partes más húmedas, se localiza en terrenos planos o con pendiente, con afloramientos de rocas calizas y suelo negro. Su estrato arbóreo es muy diversos y alcanza los $25 \mathrm{~m}$ de altura, con especies como Liquidambar styraciflua L. (alamillo), Acer skutchii Rehder (álamo plateado), Podocarpus matudae J. Buchholz \& N.E. Gray (moquito), Carya ovata var., mexicana (Engelm.) Manning (nogalillo), Cercis canadensis L. (pata de vaca) y Rhamnus capraeifolia Schltdl., (Mendiola, 2010).

Ejido San José se localiza a $99^{\circ} 13^{\prime} 46^{\prime \prime}$ latitud norte y $23^{\circ} 02$ ' 44 " longitud oeste y a una altura de 1320 msnm (Mendiola, 2010). Clima semicálido subhúmedo con lluvias en verano, salvo algunas excepciones muy locales que presentan climas templados subhúmedos. Las precipitaciones varían de los 623.8 a los $908.8 \mathrm{~mm}$ (Mendiola 2010). Se identificaron dos tipos de vegetación: bosque de pino-encino y bosque mesófilo de montaña. El bosque de pino-encino se localiza en terrenos planos o sobre laderas expuestas, suelos someros y con afloramientos rocosos en ocasiones. $\mathrm{Su}$ estrato arbóreo alcanza los $25 \mathrm{~m}$ de altura. Las especies dominantes son Pinus pseudostrobus Lindl., (pino tecatón), Quercus germana Schltdl. \& Cham., (encino roble) y Clethra pringlei S. Watson (pomarrosa). El bosque mesófilo de montaña (bosque de niebla) ocurre en cañones y zonas húmedas, frecuentemente asociado al bosque de pino-encino. El estrato arbóreo tiene aproximadamente $20 \mathrm{~m}$ de altura, con especies como Magnolia tamaulipana A. Vázquez (magnolia), Carya ovata var., mexicana (Engelm.) Manning (nogalillo), Podocarpus matudae J. Buchholz \& N.E. Gray (moquito) y Eugenia capuli (Schltdl. \& Cham.) Hook. \& Arn., (capulín) (Mendiola 2010). Se presenta el mismo tipo de suelo que en Alta Cima (Luvisol crómico, Lc) (Mendiola, 2010).

Ambas comunidades son similares en cuanto a la estructura y composición poblacional (120 en Alta Cima y 100 en San José). En ambas comunidades se presenta una mezcla heterogénea respecto al origen étnico de sus habitantes: habitantes locales y emigrantes (o sus descendientes) de los estados mexicanos de Michoacán, Hidalgo y Estado de México. Las principales actividades productivas son la agricultura de temporal: maíz (Zea mays L.), frijol (Phaseolus vulgaris L. var., jamapa), calabaza (Cucurbita pepo L.), nopal verdura (Nopalea cochinillifera (L.) Mill.) y cúrcuma ó azafrán del país (Curcuma longa L.) y el manejo de huertos familiares con frutales como durazno (Prunus persica (L.) Stokes non Batsch. var., Prisco) y guayaba criolla (Psidium guajava L.). Los principales ingresos provienen de la extracción de hojas de palmilla (Chamaedorea radicalis Mart.) y, recientemente, de la prestación de servicios ecoturísticos a través de cooperativas campesinas (Gobierno de Tamaulipas \& TNC, 2011).

Los campesinos que participaron en el estudio fueron 33 hombres y 33 mujeres (30\% de los habitantes de ambas comunidades), con promedio de edad de $45 \mathrm{y}$ 42 años, respectivamente. El $70 \%$ de ellos son oriundos de la región y han vivido toda su vida allí; el otro $30 \%$ son inmigrantes con una historia de ocupación del área de al menos 65 años. Para obtener la información en campo se realizaron entrevistas semi-estructuradas (Hoffman \& Gallaher, 2007) con el apoyo de la "ficha de encuesta etnobotánica estandarizada" según el modelo utilizado por el programa TRAMIL (Germonsen-Robineau, 1995). En la ficha de encuesta etnobotánica se solicitaron datos personales (nombre, edad, sexo, escolaridad, lugar de nacimiento propio y de sus padres, y la actividad a la que se dedica); y los relativos a las plantas útiles (nombre local, forma biológica, lugar de obtención, época de obtención, partes usadas y tipos de uso) (Anexo 1). Se corroboró la información recabada en la etapa previa con apoyo de un grupo de 10 "informantes de calidad" 
(Rodríguez-Gómez et al., 1999) a través de recorridos etnobotánicos, elaboración de "mini-herbarios" y/o registro fotográfico, materiales que se usaron para animar la discusión al final de cada sesión (Hersch \& González-Chévez, 2009). Las muestras botánicas recolectadas fueron prensadas, secadas e identificadas según la técnica estándar (Alexiades, 1996) e incorporadas al Herbario del Instituto Tecnológico de Ciudad Victoria (ITCV). Para la determinación de los ejemplares botánicos se utilizaron claves taxonómicas como Flora de Veracruz, Flora del Bajío y de Regiones Adyacentes y Flora de Guatemala, así como cotejo con los ejemplares del Herbario del Instituto Tecnológico de Ciudad Victoria. La información etnobotánica fue recopilada en tres períodos: diciembre 2012 a noviembre 2013 (encuestas etnobotánicas); diciembre 2013 a marzo 2014 (caminatas etnobotánicas) y abril a septiembre 2014 y abril a junio de 2016 (grupos focales y talleres participativos). El análisis de la información incluyó el cálculo de los siguientes índices (Toscano, 2006).

Índice de Riqueza: riqueza de conocimiento que tiene una persona sobre las posibilidades de uso de flora en su región.

$$
\mathrm{RQZ}=\frac{\text { Valor EU-Máximo }}{\Sigma \mathbf{E U}}
$$

En donde: $(R Q Z)=$ riqueza de conocimiento que tiene una persona de las especies útiles, en relación con todas las especies útiles encontradas en la región; $(E U)$ = número de especies útiles registradas por una persona; (Valor EU Máximo) = total de especies útiles reportadas en la región por todas las personas participantes en el estudio. El valor de este índice varía entre 0 y 1 , siendo 1 el valor máximo de conocimiento de la biodiversidad útil de la región.

Diversidad de uso: cuantificación del número de especies vegetales utilizadas por categorías de uso, así como evaluación de la relación de especies exógenas y especies nativas que componen cada categoría de uso. Se utiliza el índice de uso de especies exógenas (EXOG) vs., especies nativas (NAT), que hace referencia a la importancia de uso que tienen las especies nativas en relación con las especies exógenas o foráneas para cada usuario. Calculándose así:

$$
\begin{aligned}
\text { NAT } & =\frac{\Sigma \text { EU Nat }}{\Sigma \text { EU }} \\
\text { EXOG } & =\frac{\Sigma \text { EU Exog }}{\Sigma \text { EU }}
\end{aligned}
$$

En donde: $(E U N a t)=$ número de especies útiles nativas reportadas por una persona; $(E U)=$ número de especies útiles usadas por una persona; $(E U$ Exog $)=$ número de especies útiles exógenas reportadas por una persona. Estos valores se comparan para determinar cuál índice es mayor para cada persona, indicando cuales especies son las más importantes para el mismo. La suma de valores de NAT y EXOG siempre será 1 .

Para el análisis estadístico se compararon las medias del índice de riqueza en cada localidad con la prueba t de Student.

\section{Resultados.}

El total de especies vegetales útiles identificadas fue de 156 taxones. Los valores obtenidos mediante la aplicación del índice de riqueza (RQZ) no muestran diferencias significativas en el acervo de conocimiento al respecto (Tabla 1). Las familias más frecuentes en ambas comunidades fueron: Lamiaceae, Rosaceae, Asteraceae y Fabaceae.

En Alta Cima fueron identificados 117 taxones útiles, 50 de uso exclusivo y 67 con varios empleos. Las categorías de uso más frecuentes fueron: alimenticias (37\%), medicinales $(36 \%)$, ornamentales $(32 \%)$ y artesanales (14\%).

Para San José se reportaron 98 taxones útiles, 53 de uso exclusivo y 45 con varios empleos. Las categorías más demandadas fueron: medicinales (41\%), ornamentales $(35 \%)$, alimenticias $(33 \%)$ y bebidas (10\%). Las categorías de uso más demandadas en ambas comunidades fueron: alimento $(37 \%)$, medicinal $(36 \%)$, ornamental $(32 \%)$ y artesanales $(14 \%)$, mismas que engloban a las especies más importantes en la vida diaria, como medio de subsistencia y para lograr ingresos en efectivo. Las categorías de uso menos demandadas en ambas comunidades fueron: aserrío $(4 \%)$, tóxica $(1 \%)$, forrajera $(1 \%)$, veterinaria $(1 \%)$ y colorante $(0 \%)$.

Se comparte entre ambas comunidades el conocimiento sobre 60 taxones $(39 \%)$, de los cuales corresponden 45 (29\%) a especies nativas y $15(10 \%)$ a especies exógenas.

El $100 \%$ de los informantes pudieron reconocer entre el 20 y el $38 \%$ de las plantas útiles encontradas en la zona que ocupan ambas comunidades (156 especies), evidenciando un gran conocimiento etnobotánico.

Tabla 1. Prueba t de Student de las medias de plantas útiles por localidad.

\begin{tabular}{lcc}
\hline & Alta Cima & San José \\
\hline Media & 28.5 & 26.6 \\
Varianza & 22.1578947 & 11.2 \\
Observaciones & 20 & 20 \\
Varianza agrupada & 16.6789474 & \\
Diferencia hipotética de las & & \\
medias & 0 & \\
Grados de libertad & 38 & \\
Estadístico t & 1.47119175 \\
P(T<=t) una cola & 0.0747363 \\
Valor crítico de t (una cola) & 1.68595446 \\
P(T<=t) dos colas & 0.1494726 & \\
Valor crítico de t (dos colas) & 2.02439416 \\
\hline
\end{tabular}


Tabla 2. Índices de conocimiento (RQZ).

\begin{tabular}{lccccc}
\hline Comunidad & Taxones útiles & Taxones Nativos & Taxones Exógenos & $\begin{array}{c}\text { RQZ } \\
\text { Total }\end{array}$ & $\begin{array}{c}\text { \% } \\
\text { Conocimiento }\end{array}$ \\
\hline Alta Cima & 117 & 65 & 52 & 0.75 & $75 \%$ \\
San José & 98 & 42 & 59 & 0.63 & $63 \%$ \\
Ambas comunidades & 156 & 97 & 59 & & \\
\hline
\end{tabular}

En Alta Cima los usuarios conocen el $75 \%$ y en San José el $63 \%$ de las especies identificadas para la zona de estudio (Tabla 2).

Los valores promedio de conocimiento (RQZ) en ambas comunidades son similares, sin embargo se identifican los valores más altos (0.33) y más bajo (0.18) en Alta Cima. Los datos mostraron que cada persona es poseedora de una porción del conocimiento integral de la región y que, posiblemente, esté relacionado con su estrategia de vida. Los habitantes locales aún continúan apostándole a la agricultura de subsistencia y a otras alternativas de aprovechamiento integral del bosque (sobre todo de especies no maderables).

En la información proporcionada por los campesinos entrevistados hubo marcadas diferencias, el total de los informantes indicaron y usaron entre el $18 \%$ y el $34 \%$ de las plantas encontradas en la zona ocupada por ambas comunidades.

Al relacionar el índice de conocimiento (RQZ) con los rangos de edad (de los 20 años en adelante), los valores más altos se encontraron entre los usuarios de edad mediana (de 31 a 60 años), tal vez debido a que son personas que están más activas en las labores agrícolas y en el aprovechamiento del bosque, sobre todo de la palmilla (Chamaedorea radicalis).

$\mathrm{Al}$ relacionar el RQZ con los rangos de edad a nivel de ambas zonas de estudio, en Alta Cima los mayores valores de RQZ se encuentran en el grupo etario de 41 a 50 años de edad, mientras que en San José corresponden a los de mayor edad (61 y más).

Al observar lo que sucede con la riqueza de conocimientos de plantas nativas y exógenas se encuentra que en Alta Cima los que más saben de plantas nativas, pero menos de exógenas son, el grupo etario de 41 a 50 años de edad (Tabla 3).

En lo que respecta al nivel de conocimiento etnobotánico por sexo, según los datos aportados (Tabla 4), a nivel general, los hombres de Alta Cima poseen un mayor acervo de conocimiento etnobotánico que las mujeres, mientras que en San José estos valores son similares para ambos sexos.

Sin embargo, al analizar el conocimiento de especies nativas y exógenas, los habitantes de San José mostraron, para ambos sexos, RQZ más elevados sobre taxones nativos. En contraposición, en Alta Cima estos índices fueron más elevados para taxones exógenos, en hombres y mujeres.

Hay diferencias significativas en cuanto a las especies utilizadas por hombres y mujeres en ambas comunidades (Tabla 5).

\section{Discusión.}

En cuanto a la riqueza de conocimiento sobre plantas, la primera parte de la hipótesis no se comprueba al observarse un nivel de conocimiento homogéneo en los diferentes grupos etarios, sólo en la comunidad de Alta Cima existe una diferencia significativa en el grupo etario de 41 a 50 años.

La segunda parte de la hipótesis respecto a que las mujeres son las que más conocen sobre plantas, tampoco se comprobó ya que en una de las comunidades fueron los hombres (en Alta Cima) y en la otra (San José) los valores son los mismos.

Al relacionar el índice RQZ con los rangos de edad, los valores numéricos más altos se encuentran entre el grupo etario de 41 a 50 años y el grupo 61 y más, hecho que puede atribuirse a que estas personas tradicionalmente, están ligadas al aprovechamiento del bosque, especialmente a la recolección de palmilla.

Esto concuerda, parcialmente con lo identificado en comunidades campesinas donde a medida que aumenta la edad de los informantes aumenta la riqueza de conocimiento, siendo los ancianos los que mayor valoran y conocen las plantas (León et al., 2006; Ramos et al., 2007; Morales et al., 2011; Mercado, 2013).

Es importante resaltar que en los grupos etarios de jóvenes se mantienen altos valores de RQZ, lo cual contrasta con lo encontrado por autores como León et al., (2006) y Morales et al. (2011) quienes evidenciaron la pérdida parcial del conocimiento tradicional entre

Tabla 3. Grupos etarios y riqueza de conocimiento (RQZ).

\begin{tabular}{|c|c|c|c|c|c|c|c|c|c|c|c|c|}
\hline \multirow[b]{2}{*}{$\begin{array}{l}\text { Rangos } \\
\text { de } \\
\text { edad }\end{array}$} & \multicolumn{6}{|c|}{ ALTA CIMA } & \multicolumn{6}{|c|}{ SAN JOSÉ } \\
\hline & $\begin{array}{c}\# \\
\text { spp. } \\
\text { Prom }\end{array}$ & $\begin{array}{l}\text { RQZ } \\
\text { Prom }\end{array}$ & $\begin{array}{c}\# \\
\text { spp. } \\
\text { Nat }\end{array}$ & $\begin{array}{c}\text { RQZ } \\
\text { Nat }\end{array}$ & $\begin{array}{c}\# \\
\text { spp. } \\
\text { Exog }\end{array}$ & $\begin{array}{l}\text { RQZ } \\
\text { Exog }\end{array}$ & $\begin{array}{c}\# \\
\text { spp. } \\
\text { Prom }\end{array}$ & $\begin{array}{l}\text { RQZ } \\
\text { Prom }\end{array}$ & $\begin{array}{c}\# \\
\text { spp. } \\
\text { Nat }\end{array}$ & $\begin{array}{c}\text { RQZ } \\
\text { Nat }\end{array}$ & $\begin{array}{c}\# \\
\text { spp. } \\
\text { Exog }\end{array}$ & $\begin{array}{l}\text { RQZ } \\
\text { Exog }\end{array}$ \\
\hline $20-30$ & 25 & 0.21 & 11 & 0.16 & 14 & 0.27 & 26 & 0.26 & 14 & 0.33 & 12 & 0.21 \\
\hline $31-40$ & 27 & 0.23 & 16 & 0.24 & 12 & 0.22 & 0 & 0 & 0 & 0 & 0 & 0 \\
\hline $41-50$ & 31 & 0.27 & 23 & 0.35 & 8 & 0.16 & 28 & 0.28 & 20 & 0.47 & 8 & 0.14 \\
\hline $51-60$ & 27 & 0.23 & 21 & 0.32 & 6 & 0.11 & 24 & 0.24 & 14 & 0.33 & 10 & 0.18 \\
\hline $61 \mathrm{y}+$ & 29 & 0.24 & 15 & 0.23 & 14 & 0.27 & 29 & 0.29 & 18 & 0.43 & 10 & 0.19 \\
\hline
\end{tabular}

Alta Cima: 117 spp., (65 nativas y 52 exógenas); San José: 98 spp., (42 nativas y 56 exógenas). 
Tabla 4. Riqueza de conocimiento (RQZ) por sexo.

\begin{tabular}{|c|c|c|c|c|c|c|c|c|c|c|c|c|}
\hline \multirow[b]{2}{*}{$\begin{array}{l}\text { Rangos de } \\
\text { edad }\end{array}$} & \multicolumn{6}{|c|}{ HOMBRES (H) } & \multicolumn{6}{|c|}{ MUJERES (M) } \\
\hline & $\begin{array}{c}\# \\
\text { spp. } \\
\text { Prom }\end{array}$ & $\begin{array}{l}\text { RQZ } \\
\text { Prom }\end{array}$ & $\begin{array}{c}\# \\
\text { spp. } \\
\text { Nat }\end{array}$ & $\begin{array}{c}\text { RQZ } \\
\text { Nat }\end{array}$ & $\begin{array}{c}\# \\
\text { spp. } \\
\text { Exog }\end{array}$ & $\begin{array}{l}\text { RQZ } \\
\text { Exog }\end{array}$ & $\begin{array}{c}\# \\
\text { spp. } \\
\text { Prom }\end{array}$ & $\begin{array}{l}\text { RQZ } \\
\text { Prom }\end{array}$ & $\begin{array}{c}\# \\
\text { spp. } \\
\text { Nat }\end{array}$ & $\begin{array}{c}\text { RQZ } \\
\text { Nat }\end{array}$ & $\begin{array}{c}\# \\
\text { spp. } \\
\text { Exog }\end{array}$ & $\begin{array}{l}\text { RQZ } \\
\text { Exog }\end{array}$ \\
\hline Alta Cima & 29 & 0.25 & 19 & 0.29 & 10 & 0.19 & 28 & 0.24 & 16 & 0.25 & 11 & 0.22 \\
\hline San José & 27 & 0.27 & 18 & 0.44 & 8 & 0.15 & 26 & 0.27 & 15 & 0.35 & 11 & 0.20 \\
\hline
\end{tabular}

jóvenes campesinos por aculturación, migración y nula transmisión de información.

Cuando se habla de RQZ a nivel de género no hay coincidencia; en Alta Cima los hombres conocen más que las mujeres; en San José en algunos casos, las mujeres son las que poseen mayor conocimiento, por ejemplo, en plantas medicinales, alimentarias y mágico-religiosas (Toscano, 2006; Mercado, 2013; Bautista, 2015), al igual que los chamanes o curanderos (McDaniel \& Alley, 2005).

Tabla 5. Prueba $\mathrm{t}$ para dos muestras suponiendo varianzas iguales.

\begin{tabular}{|c|c|c|}
\hline & mujer & hombre \\
\hline Media & 20.66666667 & 28 \\
\hline Varianza & 53.76470588 & 11.9047619 \\
\hline Observaciones & 18 & 22 \\
\hline Varianza agrupada & 30.63157895 & \\
\hline $\begin{array}{l}\text { Diferencia } \\
\text { hipotética de las } \\
\text { medias }\end{array}$ & 0 & \\
\hline Grados de libertad & 38 & \\
\hline Estadístico t & -4.169023388 & \\
\hline $\mathrm{P}(\mathrm{T}<=\mathrm{t})$ una cola & 8.52381E-05 & $\begin{array}{l}\text { si hay significancia, } \\
\text { hay diferencias } \\
\text { entre spp., } \\
\text { utilizadas por } \mathrm{H} \text { y } \\
\text { M. }\end{array}$ \\
\hline $\begin{array}{l}\text { Valor crítico de t } \\
\text { (una cola) }\end{array}$ & 1.68595446 & \\
\hline $\mathrm{P}(\mathrm{T}<=\mathrm{t})$ dos colas & 0.000170476 & $\begin{array}{l}\text { Si hay significancia, } \\
\text { hay diferencias } \\
\text { entre spp., } \\
\text { utilizadas por H y } \\
\text { M. }\end{array}$ \\
\hline \multirow{2}{*}{$\begin{array}{l}\text { Valor crítico de t } \\
\text { (dos colas) }\end{array}$} & 2.024394164 & \\
\hline & mujer & hombre \\
\hline Media & 20.66666667 & 28 \\
\hline Error típico & 1.728273157 & 0.735612 \\
\hline Mediana & 22 & 28 \\
\hline Moda & 26 & 28 \\
\hline Desviación estándar & 7.332442014 & 3.450328 \\
\hline $\begin{array}{l}\text { Varianza de la } \\
\text { muestra }\end{array}$ & 53.76470588 & 11.90476 \\
\hline Curtosis & -1.458569948 & 0.195241 \\
\hline $\begin{array}{l}\text { Coeficiente de } \\
\text { asimetría }\end{array}$ & -0.274513672 & -0.26015 \\
\hline Rango & 21 & 15 \\
\hline Mínimo & 9 & 20 \\
\hline Máximo & 30 & 35 \\
\hline Suma & 372 & 616 \\
\hline Cuenta & 18 & 22 \\
\hline
\end{tabular}

En otros casos son los hombres los que más conocen de plantas, por ejemplo cuando se trata de plantas forestales (madera, postes, vigas, no-maderables o para aserrío) (León et al., 2006).

Respecto al conocimiento de plantas nativas $v s$. exógenas, San José muestra RQZ más elevados para especies nativas y Alta Cima para especies exógenas, tanto para hombres como para mujeres.

Algunos autores cuestionan la alta incidencia de plantas exógenas en el RQZ y atribuyen su predominancia como parte de un proceso de aculturación y un peligro para la permanencia del conocimiento tradicional, sin embargo, Bennet \& Prance (2000) lo ven como el resultado de una estrategia de diversificación, con tal de que esta incursión de nuevas especies y prácticas foráneas no sustituyan o desplacen a las nativas o redunden en homogeneización de las prácticas de manejo (McDaniel \& Alley, 2005).

\section{Conclusiones.}

Este estudio permitió comprobar que el conocimiento etnobotánico se encuentra disperso entre los diferentes grupos de edad, donde cada grupo etario es poseedor de una pequeña porción de los diversos conocimientos sobre plantas útiles en el bosque de niebla en la RBEC. Las estrategias de vida diversificada mantienen, en estas comunidades, un sistema de conocimiento más complejo y especializado, lo cual es importante para la permanencia y subsistencia de éstos grupos campesinos que deben ser considerados en el diseño y desarrollo de modelos de manejo de los recursos vegetales adaptativos con base en el conocimiento de los pobladores locales.

\section{Agradecimientos.}

Al Conacyt por el apoyo financiero a través de una beca para estudios de doctorado, y una beca para conclusión de los mismos a través del proyecto clave: CONACYT-CB-2013-01-0219979. También se agradece el apoyo de los grupos campesinos organizados y las autoridades locales en la Reserva de la Biosfera El Cielo, especialmente las de los ejidos Alta Cima y San José, Municipio de Gómez Farías, Tamaulipas, México. 
Literatura citada.

Alexiades M. 1996. Standard techniques for collecting and preparing herbarium specimens. Advances in Economic Botany vol. 10. $326 \mathrm{p}$.

Bautista R. 2015. Etnobotánica de plantas medicinales y alimenticias nativas de Mesoamérica en diez comunidades del Municipio de San Cristóbal Cucho, Departmento de San Carlos, Guatemala. Tesis PhD, Universidad de San Carlos de Guatemala. 141 p.

Bennet B. \& Prance G. 2000. Introduced plants in the indigenous pharmacopoeia of Northern South America. Economic Botany 54: 90-102.

Casas S. \& Requena G. 2005. Generalidades geográficas. En Sánchez-Ramos, G., Reyes-Castillo, P y Dirzo, R (eds.). Historia natural de la Reserva de la Biosfera El Cielo, Tamaulipas México. Universidad Autónoma de Tamaulipas. pp. 38-50.

Germonsen-Robineau L. 1995. Hacia una farmacopea caribeña. $1^{a}$ edición. Santo Domingo. Edición TRAMIL 7. $175 \mathrm{p}$.

Gobierno de Tamaulipas \& The Nature Conservancy. 2011. Plan de Conservación de la Reserva de la Biosfera El Cielo, Tamaulipas. $1^{a}$ edición. Cd. Victoria, Tam., México. Gobierno del Estado de Tamaulipas. 126 p.

González-Romo C. \& Gispert M. 2005. Etnobotánica de los solares. En: Sánchez-Ramos G. Reyes P. \& Dirzo R. (Eds). Historia Natural de la Reserva de la Biosfera El Cielo, Tamaulipas, México. Universidad Autónoma de Tamaulipas. Cd. Victoria. pp. 579-590.

Hersch P. \& González-Chévez L. 2009. Investigación participativa en etnobotánica, algunos procedimientos coadyuvantes en ella. Dimensión Antropológica 8: 129153.

Hoffman B. \& Gallaher T. 2007. Importance indices in ethnobotany. Ethnobotany Resources \& Applications 5: 201-218.

León M., Cueva P., Aguirre Z. \& Kvist L. 2006. Floristic composition, structure, endemic \& ethnobotany in the native forest "El Colorado", in Puyango, Province of Loja, Ecuador. Lyonia (10) 2: 105-115.

McDaniel J. \& Alley K. 2005. Connecting local environmental knowledge and land use practices: a human ecosystem approach to urbanization in West Georgia. Urban Ecosystems Netherlands 8: 23-38.

Mendiola A. 2010. Evaluación de la carga turística en los espacios naturales protegidos: el caso de la Reserva de la Biosfera El Cielo (Tamaulipas, México). Tesis de Doctorado en Planeación Territorial y Desarrollo Regional. Universitat de Barcelona. 129 p.

Mercado A. 2013. Estudio de las plantas medicinales usadas por los cuiicatecos en la localidad de Santos Reyes Papalo, Cuicatlán, Oax. Tesis Biología. Facultad de Ciencias. UNAM. 159 p.

Morales R., Tardío J. Aceituno L., Molina M. \& Pardo de Santayana M. 2011. Biodiversidad y Etnobotánica en España. Memorias Real Sociedad Española de Historia Natural 9: 157-207.

Pérez-Quilantán L., Mora-Olivo A. \& Medellín-Morales S. 2005. Las plantas comestibles silvestres. En: SánchezRamos G., Reyes P. \& Dirzo R. (Eds.). Historia Natural de la Reserva de la Biosfera El Cielo, Tamaulipas, México. Universidad Autónoma de Tamaulipas. Cd. Victoria, Tam., México. pp. 604-609.

Ramos M., Ávila C. \& Morales J. 2007. Etnobotánica y ecología de plantas utilizadas por tres curanderos contra la mordedura de serpiente en la región de Acayucan, Veracruz, México. Boletín Sociedad Botánica de México 81: 89-100.

Rodríguez-Gómez G., Gil J. y García-Jiménez E. 1999. Selección de Informantes y recogida de datos. En: Rodríguez-Gómez G., Gil J. y García-Jiménez E. (eds.). Metodología de la Investigación Cualitativa. Editorial Aljibe. España. pp. 135-147.

Stringer L., Dougill A., Fraser E., Hubacek K., Prell C. \& Reed M. 2006. Unpacking "participation" in the adaptive management of social-ecological systems: a critical review. Ecology and Society, Nova Scotia 11 (2), Art. 39. $14 \mathrm{p}$.

Toscano J. 2006. Uso tradicional de plantas medicinales en la vereda San Isidro, Municipio de San José de PareBoyacá: un estudio preliminar usando técnicas cuantitativas. Acta Biológica Colombiana (11) 2.10 p. 
Anexo 1. Ficha etnobotánica estandarizada

PROYECTO “CONOCIMIENTO TRADICIONAL DE LAS PLANTAS ÚTILES EN EL BOSQE DE NIEBLA DE

LA RESERVA DE LA BIOSFERA EL CIELO, TAM., MÉXICO

FORMATO DE ENCUESTA ETNOBOTÁNICA ESTANDARIZADA

Comunidad:

Sitio de Colecta:

Fecha: No. Encuesta

\begin{tabular}{|c|c|c|c|c|c|c|c|c|c|}
\hline $\begin{array}{l}\text { Nombre } \\
\text { Común }\end{array}$ & Nombre Cientifico & $\begin{array}{c}\text { Forma } \\
\text { de Vida }\end{array}$ & $\begin{array}{l}\text { Categori } \\
\text { ade } \\
\text { Uso }\end{array}$ & $\begin{array}{c}\text { Parte } \\
\text { Utilizada }\end{array}$ & Habtat & Origen & $\begin{array}{c}\text { Tipo de } \\
\text { manejo }\end{array}$ & $\begin{array}{l}\text { Como } \\
\text { se usso } \\
\text { prepara }\end{array}$ & $\begin{array}{c}\text { Tipo de } \\
\text { Manejo }\end{array}$ \\
\hline & & & & & & & & & \\
\hline & & & & & & & & & \\
\hline & & & & & & & & & \\
\hline & & & & & & & & & \\
\hline & & & & & & & & & \\
\hline & & & & & & & & & \\
\hline & & & & & & & & & \\
\hline & & & & & & & & & \\
\hline & & & & & & & & & \\
\hline & & & & & & & & & \\
\hline & & & & & & & & & \\
\hline
\end{tabular}

Datos sobre la persona que da la información

Nombre:

Edad:__ Sexo:

Dónde nació:

Tiempo de vivir en la comunidad:

cupación tiene:

Claves:

1. FORMA DE VIDA: Árbol (A); Arbusto (Ab); Hierba (Hb); Bejuco ó enredadera (Bej); Palma (Pm); Cactácea (C)

2. CATEGORÍAS DE USO: Alimenticia (Alim), Artesanía (Art), Aserrío (As), Colorante (Col), Combustible Comb), Construcción (Const), Medicinal (Med), Ornamental (Orn), Mágico-religioso (Mag-Rel) (Cond).

3. PARTE UTILIZADA: Tallo (T); Raiz (R); Hoja (H); Flor (FI); Fruto (Fr); Semilla (Se); Otros (O)

4. HABITAT (Unidad de Manejo): Solar (S); Milpa (M); Vegetación Natural (V).

5. COMO SE USA O PREPARA: Cocción (C): Infusión (I); Freído (F): Maceración (M); Otros (O)

6. MANEJO: Tolerancia (T): cuando las especies de plantas útiles o fenotipos particulares de ellas se dejan en su sitio durante los desmontes de la vegetación natural o deshierbes en los campos agrícolas; Fomento o
inducción (Fo): Comprende actividades dirigidas a incrementar la densidad poblacional y la disponibilidad de las plantas útiles mediante aclareos selectivos de la vegetación, favoreciendo algunos elementos erradicando otros, empleando riego artificial en áreas silvestres, o dispersando propágulos sexuales o vegetativos de especies o fenotipos de interés, Protección (Pr): Se lleva a cabo favoreciendo la permanencia de algunas plantas manteniéndolas bajo cuidados especiales, que pueden incluir la reducción de competencia y de herbivoros, podas, protección contra plagas, heladas, radiacion solar y sombra; Cultivo y trasplante (Cult): Incluye la siembra de semillas o plantacion de propagulos vegetativos o incluso el trasplante de individuos completos a áreas manejadas, tales como sistemas agroforestales y huertos.

7. ORIGEN: Nativa (NAT), Exógena (EXOG). 
Anexo 2. Inventario etnobotánico de Alta Cima.

\begin{tabular}{|c|c|c|c|}
\hline No. & NOMBRE COMÚN & NOMBRE CIENTÍFICO & FAMILIA \\
\hline 1 & Agapanto & Agapanthus africanus (L.) Hoffamnns. & AMARYLLIDACEAE \\
\hline 2 & Aguacate & Persea americana Mill. & LAURACEAE \\
\hline 3 & Aguacatillo & Nectandra salicifolia (Kunth) Nees & LAURACEAE \\
\hline 4 & Alamillo & Liquidambar styraciflua $\mathrm{L}$. & ALTINGIACEAE \\
\hline 5 & Albahacar & Ocimum basilicum L. & LAMIACEAE \\
\hline 6 & Alcatraz & Zantedeschia aethiopica (L.) Spreng. & ARACEAE \\
\hline 7 & Anonilla & Annona globiflora Schltdl. & ANNONACEAE \\
\hline 8 & Árbol de mora & Morus celtidifolia Kunth & MORACEAE \\
\hline 9 & Árnica & Senecio chenopodioides Kunth & ASTERACEAE \\
\hline 10 & Azafrán & Curcuma longa $\mathrm{L}$. & ZINGIBERACEAE \\
\hline 11 & Begonia & Begonia aff. heracleifolia Schltdl. \& Cham & BEGONIACEAE \\
\hline 12 & Belén & Impatiens balsamina $\mathrm{L}$. & BALSAMINACEAE \\
\hline 13 & Belén de sala & Impatiens walleriana Hook. f. & BALSAMINACEAE \\
\hline 14 & Berro & Rorippa nasturtium-aquaticum (L.) Schinz \& Thell. & BRASSICAEAE \\
\hline 15 & Calabaza & Cucurbita maxima $\mathrm{L}$. & CUCURBITACEAE \\
\hline 16 & Capulín o piste & Eugenia capuli (Schltdl. \& Cham.) Hook. \& Arn. & MYRTACEAE \\
\hline 17 & Cedro rojo & Cedrela odorata $\mathrm{L}$ & MELIACEAE \\
\hline 18 & Cedro chino & Juniperus deppeana Steud. & CUPRESSACEAE \\
\hline 19 & Chamalillo & Ceratozamia kuesteriana Regel & ZAMIACEAE \\
\hline 20 & Chayote & Sechium edule Sw. & CUCURBITACEAE \\
\hline 21 & Cordoncillo & Piper amalago L. & PIPERACEAE \\
\hline 22 & Coyolillo & Canna indica $\mathrm{L}$. & CANNACEAE \\
\hline 23 & Cuchillitos o colorín & Erythrina americana Mill. & FABACEAE \\
\hline 24 & Durazno & Prunus persica (L.) Batsch var. prisco & ROSACEAE \\
\hline 25 & Encino blanco & Quercus polymorpha Schltdl. \& Cham. & FAGACEAE \\
\hline 26 & Encino rojo & Quercus germana Schltdl. \& Cham. & FAGACEAE \\
\hline 27 & Epazote & Dysphania ambrosioides (L.) Mosyakin \& Clemants & AMARANTHACEAE \\
\hline 28 & Frijol negro & Phaseolus vulgaris L. var. negro & FABACEAE \\
\hline 29 & Gladiola & Gladiolus communis L. & IRIDACEAE \\
\hline 30 & Guaco & Cissampelos pareira $\mathrm{L}$. & MENISPERMACEAE \\
\hline 31 & Guaje & Leucaena leucocephala (Lam.) de Wit & FABACEAE \\
\hline 32 & Guajillo o tepeguaje & Leucaena pulverulenta (Schltdl.) Benth. & FABACEAE \\
\hline 33 & Guayaba & Psidium guajava $\mathrm{L}$. & MYRTACEAE \\
\hline 34 & Hierbabuena & Mentha spicata $\mathrm{L}$. & LAMIACEAE \\
\hline 35 & Higo & Ficus carica L. & CARICACEAE \\
\hline 36 & Higuerilla & Ricinus communis $L$. & EUPHORBIACEAE \\
\hline 37 & Hortensia & Hydrangea macrophylla (Thunb.) Ser. & HYDRANGEACEAE \\
\hline 38 & Hueso de tigre & Wimmeria concolor Schltdl. \& Cham. & CELASTRACEAE \\
\hline 39 & Huizache & Acacia farnesiana (L.) Willd. & FABACEAE \\
\hline 40 & $\begin{array}{l}\text { Injerto rojo o } \\
\text { cuasilaca }\end{array}$ & Psittacanthus calyculatus (DC.) G. Don & LORANTHACEAE \\
\hline 41 & Istafiate & Artemisia ludoviciana Nutt. subsp. mexicana (Willd.) Keck & ASTERACEAE \\
\hline 42 & Jaboncillo & Sapindus saponaria $\mathrm{L}$. & SAPINDACEAE \\
\hline 43 & Jabonero o cóngora & Phytolacca icosandra L. & PHYTOLACCACEAE \\
\hline 44 & Jacubo & Acanthocereus tetragonus (L.) Hummerlinck & CACTACEAE \\
\hline 45 & Juanjilón & Pseudobombax ellipticum (Kunth) Dugand & MALVACEAE \\
\hline 46 & Laurel & Litsea glaucescens Kunth & LAURACEAE \\
\hline 47 & Limón dulce & Citrus limetta Risso & RUTACEAE \\
\hline 48 & Limoncillo & Esenbeckia berlandieri Baillon & RUTACEAE \\
\hline 49 & Lirio rojo & Hippeastrum hybrid & AMARYLLIDACEAE \\
\hline 50 & $\begin{array}{l}\text { Lirio blanco en } \\
\text { racimo }\end{array}$ & Crinum commelynii Jacq. & AMARYLLIDACEAE \\
\hline 51 & Lirio rosa en racimo & Crinum asiaticum $\mathrm{L}$. & AMARYLLIDACEAE \\
\hline 52 & Magnolia & Magnolia tamaulipana A. Vázquez & MAGNOLIACEAE \\
\hline 53 & Maíz & Zea mays L. & POACEAE \\
\hline 54 & Malva babosa & Heliocarpus donell-smithii Rose & MALVACEAE \\
\hline 55 & Mandarina & Citrus reticulata Blanco & RUTACEAE \\
\hline 56 & Mariposa & Hedychium coronarium J. Koenig & ZINGIBERACEAE \\
\hline 57 & Maravilla & Mirabilis longiflora $\mathrm{L}$. & NYCTAGINACEAE \\
\hline 58 & Mejorana & Origanum majorana $\mathrm{L}$. & LAMIACEAE \\
\hline 59 & Mirto & Salvia microphylla Kunth & LAMIACEAE \\
\hline
\end{tabular}


Anexo 2. Inventario etnobotánico de Alta Cima.

\begin{tabular}{|c|c|c|c|}
\hline No. & NOMBRE COMÚN & NOMBRE CIENTÍFICO & FAMILIA \\
\hline 60 & Monilla o cascabelito & Ungnadia speciosa Endl. & SAPINDACEAE \\
\hline 61 & Mora & Chlorophora tinctoria (L.) Gaudich. ex Benth. & MORACEAE \\
\hline 62 & Muicle & Justicia spicigera Schltdl. & ACANTHACEAE \\
\hline 63 & Naranja & Citrus sinensis L. var. Valencia & RUTACEAE \\
\hline 64 & Naranjillo & Trichillia havanensis Jacq. & MELIACEAE \\
\hline 65 & Naranjita japonesa & Fortunella margarita (Lour.) Swingle. & RUTACEAE \\
\hline 66 & Níspero & Eryobotria japonica (Thunb.) Lindl. & ROSACEAE \\
\hline 67 & Nogalillo & Carya ovata var. mexicana (Engelm.) Manning & JUGLANDACEAE \\
\hline 68 & Nogal cimarrón & Juglans mollis Engelm. & JUGLANDACEAE \\
\hline 69 & Nopal verdulero & Nopalea cochenillifera (L.) Salm-Dyck & CACTACEAE \\
\hline 70 & Nogal encarcelado & Carya illinoinensis (Wangenh.) K. Koch & JUGLANDACEAE \\
\hline 71 & Aquiche o guácima & Guazuma ulmifolia Lam. & MALVACEAE \\
\hline 72 & Ojite & Brosimum alicastrum $\mathrm{Sw}$. & MORACEAE \\
\hline 73 & $\begin{array}{l}\text { Horcajuda o } \\
\text { hediondilla }\end{array}$ & Cestrum dumetorum Schltdl. & SOLANACEAE \\
\hline 74 & Orégano hoja chica & Lippia graveolens Kunth. & LAMIACEAE \\
\hline 75 & Orquídea blanca & Euchile mariae (Ames) Withner & ORCHIDACEAE \\
\hline 76 & Orquídea calavera & Stanhopea tigrina Bateman ex Lindl & ORCHIDACEAE \\
\hline 77 & Orquídea pasto & Isochilus unilateralis B.L. Rob. & ORCHIDACEAE \\
\hline 78 & $\begin{array}{l}\text { Orquídea negra o } \\
\text { pulpito }\end{array}$ & Prosthechea cochleata (L.) W.E. Higgins & ORCHIDACEAE \\
\hline 79 & Zapatito zapatito & Lycaste deppei (Lodd.) Lindl. & ORCHIDACEAE \\
\hline 80 & Pagüilla o pomarrosa & Clethra pringlei S.Watson & CLETHRACEAE \\
\hline 81 & Palmilla & Chamaedorea radicalis Mart. & ARECACEAE \\
\hline 82 & $\begin{array}{l}\text { Palo bolero o palo } \\
\text { amarillo }\end{array}$ & Rhamnus capreifolia Schltdl. & RHAMNACEAE \\
\hline 83 & $\begin{array}{l}\text { Palo de agua o palo } \\
\text { santo }\end{array}$ & Dendropanax arboreus (L.) Decne \& Planch & ARALIACEAE \\
\hline 84 & Papaya cimarrona & Vasconcellea cauliflora (Jacq.) A. DC. & CARICACEAE \\
\hline 85 & $\begin{array}{l}\text { Papayilla, llora sangre } \\
\text { o calderona }\end{array}$ & Bocconia frutescens $\mathrm{L}$. & PAPAVERACEAE \\
\hline 86 & Pata de vaca & Cercis canadensis $\mathrm{L}$. & FABACEAE \\
\hline 87 & Pasionaria & Passiflora conzattiana Killip & PASSIFLORACEAE \\
\hline 88 & Pino nylon o triste & Pinus patula Schltdl. \& Cham. & PINACEAE \\
\hline 89 & Pino tecatón & Pinus pseudostrobus Lindl. & PINACEAE \\
\hline 90 & Pohua & Trema micrantha (L.) Blume & CANNABACEAE \\
\hline 91 & Quelite blanco & Amaranthus hybridus L. & AMARANTHACEAE \\
\hline 92 & $\begin{array}{l}\text { Quelite blanco o } \\
\text { quintonil }\end{array}$ & Amaranthus palmeri S. Watson & AMARANTHACEAE \\
\hline 93 & Quelite morado & Solanum nigrescens $\mathrm{M}$. Martens \& Galeotti & SOLANACEAE \\
\hline 94 & $\begin{array}{l}\text { Rejalgar u oreja de } \\
\text { elefante }\end{array}$ & Xanthosoma robustum Schott & ARACEAE \\
\hline 95 & Romero & Rosmarinus officinalis L. & LAMIACEAE \\
\hline 96 & Romerillo & Taxus globosa Schltdl. & TAXACEAE \\
\hline 97 & Rosa de castilla & Rosa centifolia $\mathrm{L}$. & ROSACEAE \\
\hline 98 & Ruda & Ruta graveolens $\mathrm{L}$. & RUTACEAE \\
\hline 99 & Salvia & Salvia gesneriflora Lindl. \& Paxton & LAMIACEAE \\
\hline 100 & San Pedro o tronadora & Tecoma stans (L.) Juss. ex Kunth. & BIGNONACEAE \\
\hline 101 & Talayote & Marsdenia coulteri Hemsl. & APOCYNACEAE \\
\hline 102 & Té huasteco & Bidens squarrosa Kunth. & ASTERACEAE \\
\hline 103 & Tejocote cimarrón & Crataegus rosei Eggl & ROSACEAE \\
\hline 104 & Tila & Tilia houghi Rose & TILIACEAE \\
\hline 105 & Tostada de caballo & Petrea volubilis $\mathrm{L}$. & VERBENACEAE \\
\hline 106 & $\begin{array}{l}\text { Chacloco, tres hojitas } \\
\text { o madura plátano }\end{array}$ & Hamelia patens Jacq. & RUBIACEAE \\
\hline 107 & $\begin{array}{l}\text { Trompeta de ángel o } \\
\text { floripondio }\end{array}$ & Brugmansia suaveolens (Humb. \& Bonpl. ex Willd.) Sweet & SOLANACEAE \\
\hline 108 & Trompillo o tila & Ternstroemia sylvatica Schltdl. \& Cham. & VITACEAE \\
\hline 109 & Uva de monte & Vitis cinerea (Engelm.) Millardet & VITACEAE \\
\hline 110 & Uva de monte & Vitis tiliifolia Humb. \& Bonpl. ex Schult. & VITACEAE \\
\hline 111 & Venadilla o tepehua & Porophyllum macrocephalum DC. & ASTERACEAE \\
\hline
\end{tabular}


Anexo 2. Inventario etnobotánico de Alta Cima.

\begin{tabular}{llll}
\hline No. & NOMBRE COMÚN & NOMBRE CIENTÍFICO & FAMILIA \\
\hline 112 & Venadilla o tepehua & Porophyllum ruderale (Jacq.) Cass. & ASTERACEAE \\
& morada & & \\
113 & Verdolaga & Portulaca oleracea L. & PORTULACACEAE \\
114 & Zacate limón & Cymbopogon citratus (DC.) Stapf & POACEAE \\
115 & Zarza & Rubus sapidus Schltdl. & ROSACEAE \\
116 & Zarza & Rubus coriifolius Liebm. & ROSACEAE \\
117 & Zarzaparrilla & Smilax bona-nox L. & SMILACACEAE \\
\hline
\end{tabular}

Anexo 3. Inventario etnobotánico de San José.

\begin{tabular}{|c|c|c|c|}
\hline No. & NOMBRE COMÚN & NOMBRE CIENTÍFICO & FAMILIA BOTÁNICA \\
\hline 1 & Acelga & Beta vulgaris subsp. cicla (L.) W.D.J. Koch & AMARANTHACEAE \\
\hline 2 & Aguacatillo & Nectandra salicifolia (Kunth) Nees & LAURACEAE \\
\hline 3 & Ajenjo & Artemisia absinthium L. & ASTERACEAE \\
\hline 4 & Alamillo & Liquidambar styraciflua $\mathrm{L}$. & ALTINGIACEAE \\
\hline 5 & Albahacar & Ocimum basilicum $\mathrm{L}$. & LAMIACEAE \\
\hline 6 & Alcatraz & Zantedeschia aethiopica (L.) Spreng. & ARACEAE \\
\hline 7 & Andrina o ciruela & Prunus domestica L. & ROSACEAE \\
\hline 8 & Anisillo o pericón & Tagetes lucida Cav. & ASTERACEAE \\
\hline 9 & Anonilla & Annona globiflora Schltdl. & ANNONACEAE \\
\hline 10 & Begonia & Begonia spp. & BEGONIACEAE \\
\hline 11 & Berro & Rorippa nasturtium-aquaticum (L.) Schinz \& Thell. & BRASSICAEAE \\
\hline 12 & Calabaza & Cucurbita maxima $\mathrm{L}$. & CUCURBITACEAE \\
\hline 13 & Capulín & Prunus serotina subsp. capuli (Cav.) McVaugh & ROSACEAE \\
\hline 14 & Cedro chino & Juniperus deppeana Steud. & CUPRESSACEAE \\
\hline 15 & Cedro rojo & Cedrela odorata $\mathrm{L}$. & MELIACEAE \\
\hline 16 & Chaya & Cnidoscolus chayamansa $\mathrm{McVaugh}$ & EUPHORBIACEAE \\
\hline 17 & Chayote & Sechium edule Sw. & CUCURBITACEAE \\
\hline 18 & Chile payaso & Capsicum annum hybrid "NuMex Twilight" & SOLANACEAE \\
\hline 19 & Chipús & Asclepias curassavica $\mathrm{L}$. & ASCLEPIADACEAE \\
\hline 20 & Cigarrilla & Decatropis bicolor (Zucc.) Radlk. & RUTACEAE \\
\hline 21 & Cuasia o palo amarillo & Berberis gracilis Benth. var. madrensis Marroq. & BERBERIDACEAE \\
\hline 22 & Dalia & Dahlia hybrid Cav. & ASTERACEAE \\
\hline 23 & Dalia sencilla & Dahlia imperialis Roezl ex Ortgies & ASTERACEAE \\
\hline 24 & Dalia silvestre & Dalia coccinea Cav. & ASTERACEAE \\
\hline 25 & Durazno & Prunus persica (L.) Batsch var. Prisco & ROSACEAE \\
\hline 26 & Encino blanco & Quercus polymorpha Schltdl. \& Cham. & FAGACEAE \\
\hline 27 & Encino roble o rojo & Quercus germana Schltdl. \& Cham. & FAGACEAE \\
\hline 28 & Eucalipto & Eucalyptus globulus Labill. & MIRTACEAE \\
\hline 29 & Floripondio & Brugmansia candida Pers. & SOLANACEAE \\
\hline 30 & Frijol negro & Phaseolus vulgaris L. var. Negro & FABACEAE \\
\hline 31 & Gallitos & Catopsis nutans (Sw.) Griseb. & BROMELIACEAE \\
\hline 32 & Gardenia & Gardenia jasminoides J. Ellis & RUBIACEAE \\
\hline 33 & Gladiola & Gladiolus communis L. & IRIDACEAE \\
\hline 34 & Guaje & Leucaena leucocephala (Lam.) de Wit & FABACEAE \\
\hline 35 & Guajillo o tepeguaje & Leucaena pulverulenta (Schltdl.) Benth. & FABACEAE \\
\hline 36 & Guayaba & Psidium guajava $\mathrm{L}$. & MYRTACEAE \\
\hline 37 & Hierbabuena & Mentha spicata $\mathrm{L}$. & LAMIACEAE \\
\hline 38 & Hierba del burro o & Eupatorium pycnocephalum Less. & ASTERACEAE \\
\hline 39 & Hierba del golpe & Oenothera rosea L'Hér. ex Aiton & ONAGRACEAE \\
\hline 40 & Hinojo & Foeniculum vulgare Mill. & APIACEAE \\
\hline 41 & Hortensia & Hydrangea macrophylla (Thunb.) Ser. & HYDRANGEACEAE \\
\hline 42 & Istafiate & Artemisia ludoviciana Nutt. subsp. mexicana (Willd.) Keck & ASTERACEAE \\
\hline 43 & Jabonero o cóngora & Phytolacca icosandra $\mathrm{L}$. & PHYTOLACCACEAE \\
\hline 44 & Laurel & Litsea glaucescens Kunth & LAURACEAE \\
\hline 45 & Lirio rojo & Hippeastrum hybrid & AMARYLLIDACEAE \\
\hline 46 & Lirio blanco en racimo & Crinum commelynii Jacq. & AMARYLLIDACEAE \\
\hline
\end{tabular}


Anexo 3. Inventario etnobotánico de San José.

\begin{tabular}{|c|c|c|c|}
\hline No. & NOMBRE COMÚN & NOMBRE CIENTÍFICO & FAMILIA BOTÁNICA \\
\hline 47 & Lirio rosa en racimo & Crinum asiaticum $\mathrm{L}$. & AMARYLLIDACEAE \\
\hline 48 & Magnolia & Magnolia tamaulipana A. Vázquez & MAGNOLIACEA \\
\hline 49 & Maíz & Zea mays $\mathrm{L}$. & POACEAE \\
\hline 50 & Mala mujer & Cnidoscolus multilobus (Pax) I. M. Johnston & EUPHORBIACEAE \\
\hline 51 & Manzanilla & Matricaria chamomilla $\mathrm{L}$. & ASTERACEAE \\
\hline 52 & Mirto & Salvia microphylla Kunth & LAMIACEAE \\
\hline 53 & Moquito & Podocarpus matudae Lundell & PODOCARPACEAE \\
\hline 54 & Mora & Chlorophora tinctoria (L.) Gaudich. ex Benth. & MORACEAE \\
\hline 55 & Níspero & Eryobotria japonica (Thunb.) Lindl. & ROSACEAE \\
\hline 56 & Nopal tunero & Opuntia ficus-indica (L.) Miller & CACTACEAE \\
\hline 57 & Nopal verdulero & Nopalea cochenillifera (L.) Salm-Dyck & CACTACEAE \\
\hline 58 & Nogal cimarrón & Juglans mollis Engelm. & JUGLANDACEAE \\
\hline 59 & Horcajuda o hediondilla & Cestrum dumetorum Schltdl. & SOLANACEAE \\
\hline 60 & Orquídea zapatito & Lycaste deppei (Lodd.) Lindl. & ORCHIDACEAE \\
\hline 61 & Orquídea blanca & Euchile mariae (Ames) Withner & ORCHIDACEAE \\
\hline 62 & Orquídea calavera & Stanhopea tigrina Bateman ex Lindl & ORCHIDACEAE \\
\hline 63 & Oyamel & Abies vejari Martínez & PINACEAE \\
\hline 64 & Pagüilla o pomarrosa & Clethra pringlei S. Watson & CLETHRACEAE \\
\hline 65 & Palmilla & Chamaedorea radicalis Mart. & ARECACEAE \\
\hline 66 & Palo bolero o amarillo & Rhamnus capreifolia Schltdl. & RHAMNACEAE \\
\hline 67 & Pata de vaca & Cercis canadensis $\mathrm{L}$. & FABACEAE \\
\hline 68 & Paxtle & Tillandsia usneoides (L.) L. & BROMELIACEAE \\
\hline 69 & Pera & Pyrus communis L. & ROSACEAE \\
\hline 70 & Pino nylon o triste & Pinus patula Schltdl. \& Cham. & PINACEAE \\
\hline 71 & Pino tecatón & Pinus pseudostrobus Lindl. & PINACEAE \\
\hline 72 & Pohua & Trema micrantha (L.) Blume & CANNABACEAE \\
\hline 73 & $\begin{array}{l}\text { Quelite blanco o } \\
\text { quintonil }\end{array}$ & Amaranthus palmeri S. Watson & AMARANTHACEAE \\
\hline 74 & Quelite morado & Solanum nigrescens $\mathrm{M}$. Martens \& Galeotti & SOLANACEAE \\
\hline 75 & Quelite de pata roja & Amaranthus spp. & AMARANTHACEAE \\
\hline 76 & Real de oro & Achillea millefolium $\mathrm{L}$. & ASTERACEAE \\
\hline 77 & $\begin{array}{l}\text { Rejalgar u oreja de } \\
\text { elefante }\end{array}$ & Xanthosoma robustum Schott & ARACEAE \\
\hline 78 & Romero & Rosmarinus officinalis $\mathrm{L}$. & LAMIACEAE \\
\hline 79 & Romerillo & Taxus globosa Schltdl. & TAXACEAE \\
\hline 80 & Ruda & Ruta graveolens $\mathrm{L}$. & RUTACEAE \\
\hline 81 & Sábila & Aloe vera $\mathrm{L}$. & ASPHODELACEAE \\
\hline 82 & Sauco & Sambucus nigra subsp. canadensis (L.) Bolli & ADOXACEAE \\
\hline 83 & Siete negritos & Lantana hirta Graham & VERBENACEAE \\
\hline 84 & Tejocote cimarrón & Crataegus rosei Eggl. & ROSACEAE \\
\hline 85 & Tila & Tilia houghi Rose & TILIACEAE \\
\hline 86 & Toloache & Datura inoxia Mill. & SOLANACEAE \\
\hline 87 & Tomillo & Thymus vulgaris $\mathrm{L}$. & LAMIACEAE \\
\hline 88 & Toronjil & Agastache mexicana (Kunth) Lint \& Epling & LAMIACEAE \\
\hline 89 & $\begin{array}{l}\text { Trompeta de ángel, } \\
\text { campana o floripondio }\end{array}$ & Brugmansia suaveolens (Humb. \& Bonpl. ex Willd) Sweet & SOLANACEAE \\
\hline 90 & Trompillo o tila & Ternstroemia sylvatica Schltdl. \& Cham. & THEACEAE \\
\hline 91 & Tulipán & Hibiscus rosa-sinensis $\mathrm{L}$. & MALVACEAE \\
\hline 92 & Uva de monte & Vitis cinerea (Engelm.) Millardet & VITACEAE \\
\hline 93 & Uva de monte & Vitis tiliifolia Humb. \& Bonpl. ex Schult. & VITACEAE \\
\hline 94 & $\begin{array}{l}\text { Vara de San José o } \\
\text { Nardo }\end{array}$ & Polianthes tuberosa $\mathrm{L}$. & AGAVACEAE \\
\hline 95 & Verdolaga & Portulaca oleracea $\mathrm{L}$. & PORTULACACEAE \\
\hline 96 & Zacate limón & Cymbopogon citratus (DC.) Stapf & POACEAE \\
\hline 97 & Zarza & Rubus sapidus Schltdl. & ROSACEAE \\
\hline 98 & Zarza & Rubus coriifolius Liebm. & ROSACEAE \\
\hline
\end{tabular}


${ }^{1}$ Profesor de carrera. Licenciatura en Nutrición. Universidad Autónoma de Tamaulipas. Unidad Académica Trabajo Social y Ciencias para el Desarrollo Humano. sgmede@ gmail.com. +52-(834)-155-89-79.

${ }^{2}$ Profesora-investigadora. División de Estudios de Posgrado e investigación. Instituto Tecnológico de Ciudad Victoria. Blvd. Emilio Portes Gil No. 1301. Ciudad Victoria, Tamaulipas, C.P. 87010. ludivinab@yahoo.com; +52-(834)-207-96-39.

${ }^{3}$ Instituto de Ecología Aplicada, Universidad Autónoma de Tamaulipas. amorao@uat.edu.mx.

${ }^{4}$ Profesor-investigador. División de Estudios de Posgrado e investigación. Instituto Tecnológico de Ciudad Victoria. Blvd. Emilio Portes Gil No. 1301. Ciudad Victoria, Tamaulipas, C.P. 87010. almagavetec@ hotmail.com; +52-(811)-628-48-24.

${ }^{5}$ Investigadora. Instituto de Ecología Aplicada. Universidad Autónoma de Tamaulipas. Calle Division del Golfo 356. Colonia Libertad. Ciudad Victoria 87019. sgmoravelo@ hotmail.com; +52-(551)-321-37-40. 